\title{
Decision Trees based Fuzzy Rules
}

\author{
Mohammed A. Al-Gunaid ${ }^{\mathrm{a}}$, Maxim V. Shcherbakov ${ }^{\mathrm{a}}$, Valeriy A. Kamaev ${ }^{\mathrm{a}}$, Olga M. Gerget ${ }^{\mathrm{b}}$, Anton P. Tyukov ${ }^{\mathrm{a}}$ \\ ${ }^{a}$ Volgograd State Techincle University, Volgograd, Russia \\ ${ }^{\mathrm{b}}$ National Research Tomsk Polytechnic University, Tomsk, Russia
}

mohammadalgunaid@gmail.com

\begin{abstract}
Decision trees have been recognized as interpretable, efficient, problem independent and scalable architectures. In case of fuzzy representation there is no procedure of automation tree building. In other words existing approaches of building decision trees and fuzzy decision trees cannot provide automatically generate fuzzy sets and fuzzy knowledge bases to build fuzzy decision trees. Paper presents a new method of building fuzzy decision trees called decision trees based fuzzy rules (DTFR). This method combines tree growing and pruning, to determine the structure of the FDT, to improve its generalization capabilities. Proposes a method (DTFR) considered as a variant of decision tree inductive using fuzzy set theory.
\end{abstract}

Keywords: Decision tree; Fuzzy logic; Forecasting; Classification; Inductive learning.

\section{INTRODUCTION}

Decision trees (DT) have been recognized as interpretable, efficient, problem independent and scalable architectures. There are several reasons behind their visibility and broad applicability. First, in many cases the accuracy of decision trees is comparable or higher than the accuracy of other classification models [1]. Second, most decision trees do not require a large number of parameters to be adjusted in their design [2]. Third, due to their intuitively appealing topology, the resulting classification models become easy to comprehend [3][4]. The perfect data mining technique would simultaneously: be efficient when training and using it, be a problem-independent tool that manages to automatically extract the most relevant features for a given problem and to fix its parameters and its model complexity with minimal human intervention [5].

Decision tree is useful for discovering hidden rules when dealing with a large amount of data [6]. However, decision trees could be highly unstable classifiers with respect to noise present in the training data. They are also recognized as highly unstable classifiers with respect to minor perturbations in the training data, in other words, methods presenting high variance. Fuzzy sets and fuzzy arithmetic provide a vehicle to use linguistic and qualitative features to form rules that are more understandable and accurate [7].

In light of these observations, we can draw a conclusion that existing approaches of building DT and FDT cannot provide automatically generate fuzzy sets and fuzzy knowledge bases (FKB) to build FDT. In this paper proposes a method called decision trees based fuzzy rules (DTFR), i.e. a variant of decision tree inductive using fuzzy set theory. In this research, a decision tree tool ID3 (Iterative Dichotomizer 3) [8] is combined fuzzy with fuzzy theory to develop an clustering fuzzy decision tree for time series decision. The proposed method is able to predict the time series more precisely and to offer speculators a better information platform.

\section{RELATED WORKS}

The decision trees are one of the most popular machinelearning techniques [9][10] due to their ability and ease of use for supporting human decision making in an easy to understand and complete form [6][11]. The applied literature of FDT to problems is considerably less than standard decision trees. It is a relatively new domain and much focus has been on further refining the algorithm rather than exploring alternative applications [12][13]. FDT are a powerful search methodology that combines symbolic tree structures with approximate reasoning offered by fuzzy representation [14].

FDT applications within the literature include a weighted approach to the prognosis of breast-cancer survivability [15] which were proven to be a strong classifier and predictor real world decision applications. In the work [16] applied FDT to the context of power security assessment and were particularly beneficial for handling large scale problems whilst combining interpretability with smooth interpolation capabilities. The recently proposed a FDT based method for spam behavior recognition [17]. The knowledge of the FDT detected and classified spam sender behavior patterns by analyzing them automatically.

In this case, oblique decision trees are more suitable. Most oblique decision trees are associated with a linear decision function positioned at each node [18][3][19]. However, oblique trees are difficult to interpret. In the work [20] discusses that most kind of decisions can be made only by using well known 'if-then-else' structure. However, it is rather difficult to write such language rules when a large number of conditions would exist. A solution to this is to use systems which rely on welldefined data to create these knowledge rules. Since numerous attributes are considered when evaluating the suitability of a manufacturing technology, most information available is subjective and imprecise [21] and therefore modeling the vagueness through a form of fuzzy logic is appropriate. The advantage of solving a problem defined through fuzzy data sets for decision problems is that they are able to provide a basis of analysing historical data decision information [22]. 


\section{METHODOLOGY}

\section{A. Data fuzzification}

This research applies fuzzy resolution concept in fuzzy set theory to transform data attribute from continuous to discrete [23]. It also utilizes decision tree classification method to build a forecasting model.

As a rule, the formation fuzzy variables are carried out by the experts or users, who deciding the task such as forecasting time series. There are two reasons to use algorithm of the formation fuzzy variables based time series: i) if the problem is solved by people without special training, it is quite difficult to identify a set of fuzzy sets associated with variables and set their parameters, ii) it also difficult to formation fuzzy sets based time series, when we have a big data.

Researchers always consider different problems with different membership function; the most used membership function includes Triangles membership functions, trapezoid membership functions, and Gauss membership functions [24]. This research will adopt Gauss membership functions for our primary membership functions.

Let there be a time series $\left\{x_{i}\right\}$. Assume that the current value depends on the previous values of time series i.e. process is described by autoregressive model. We will consider that all of the variables used to construct the autoregressive model and we can define fuzzy sets $\left\{A_{j}\right\}$, where $j=\overline{1, m_{A}}$, which defines membership function. Consequently, we need to obtain a set of fuzzy sets $\left\{A_{j}\right\}$ and kind of Gaussian membership functions $\mu_{A}\left(x ; c_{j}, \sigma_{j}\right)$, where $\mathrm{j}$ - number of fuzzy sets, $c_{j}-$ center of fuzzy sets $\left\{A_{j}\right\}, \sigma_{j}-$ width of Gaussian membership functions. The Gaussian membership functions are shown in Fig. 1.

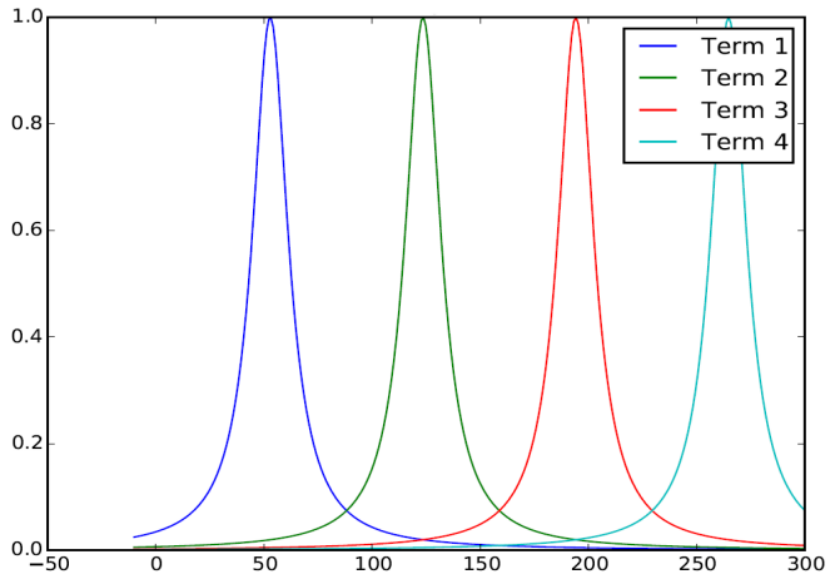

Fig. 1. Example of Gaussian membership functions.

The value of fuzzy sets is calculated as follows:

$$
\mu_{\mathrm{A}}\left(\mathrm{x} ; \mathrm{c}_{\mathrm{j}}, \sigma_{\mathrm{j}}\right)=\frac{1}{1+\left(\frac{\mathrm{x}_{\mathrm{i}}-\mathrm{b}_{\mathrm{j}}}{\sigma_{\mathrm{j}}}\right)^{2}},
$$

Where $x_{j}-$ the element of time series. Centers classes of fuzzy sets will be defined by the following equation:

$$
c_{j}=x_{\min }+2 j \Delta c \text {, }
$$

Where $x_{\min }-$ minimum value of the time series $\left\{x_{i}\right\}$, $\Delta c$ - step obtained in accordance with the formula:

$$
\Delta c=\frac{x_{\max }-x_{\min }}{m_{A}}
$$

Where $x_{\max }-$ maximum value of the time series $\left\{x_{i}\right\}$.

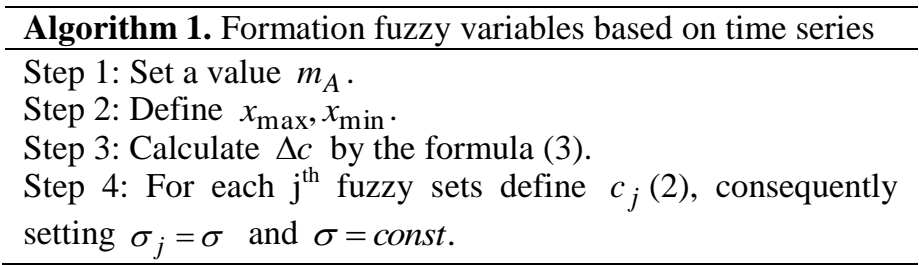

\section{B. Fuzzy knowledge base}

Under the fuzzy knowledge base (FKB) understood as a set of fuzzy production rules. The main idea of the proposed below approach is i) exclusion of experts from the procedure of formation of fuzzy rules as a result of time series analysis, ii) automatically generate fuzzy knowledge base [25][26].

\section{Algorithm 2. Formation fuzzy knowledge base \\ Input: Fuzzy time series $\left\{A_{k}^{(i)}\right\}$ \\ Output: Rules $r_{l}$ obtained from the left and right term FKB}

1. Set $n$ autoregressive variable and value of $\log \tau$.

2 . Set the current value of time $t=\tau n+1$.

3. Choose value from time series $\tilde{X}(t)=\left\{x_{t}-i \tau\right\}$, where $i=\overline{1, n}$

4. Generate the left term of the FKB

a) For each value from $\left\{x_{t}-i \tau\right\}$ choose fuzzy sets $\left\{A_{k}^{(i)}\right\}, k=\overline{1, n}$, where

$$
A_{k}^{(i)}=\arg \left(\max _{k=1, m_{A}}\left(\mu_{A_{t}}\left(x_{i}\right)\right)\right),
$$

i.e. fuzzy sets for which defined the minimum value of the membership.

b) Create $n$ terms $x_{t}-i \tau=A_{k}^{(i)}$, where $i=\overline{1, n}$.

c) Create the left side of the conjunction rules.

5. Generate right term of the FKB for $x_{t}$ similarly 4 .

6. Create rules $r_{l}$ from obtained left and right terms.

7. Check if there is a rule $r_{l}$. If not a rule then add to FKB.

8 . If the condition $t=t_{k}$, then end the procedure.

ELSE

Setting $t=t+1$ and go to step 3 .

\section{A forecasting model by fuzzy decision trees based fuzzy rules}

After transforming numerical data into fuzzy set data, next step will be to classify the data by ID3 decision tree for future time series prediction. ID3 decision tree is based on Information Gain, which means chaos of data. The higher the Gain value, the more chaos the data is. As a result, the biggest information gain is selected as an attribute value. Then, the 
decision tree use this attribute value splitting data for more training sets, those training sets repeatedly attempt to search for the biggest information gain until no more splitting [24].

First suppose training data set $S$ have $m$ categories, it means

$$
E(A)=\sum_{j=1}^{v} \frac{s_{i j}+\ldots+s_{m j}}{S} \cdot I\left(s_{i j}, \ldots, s_{m j}\right)
$$

The detailed procedures of method decision trees based fuzzy
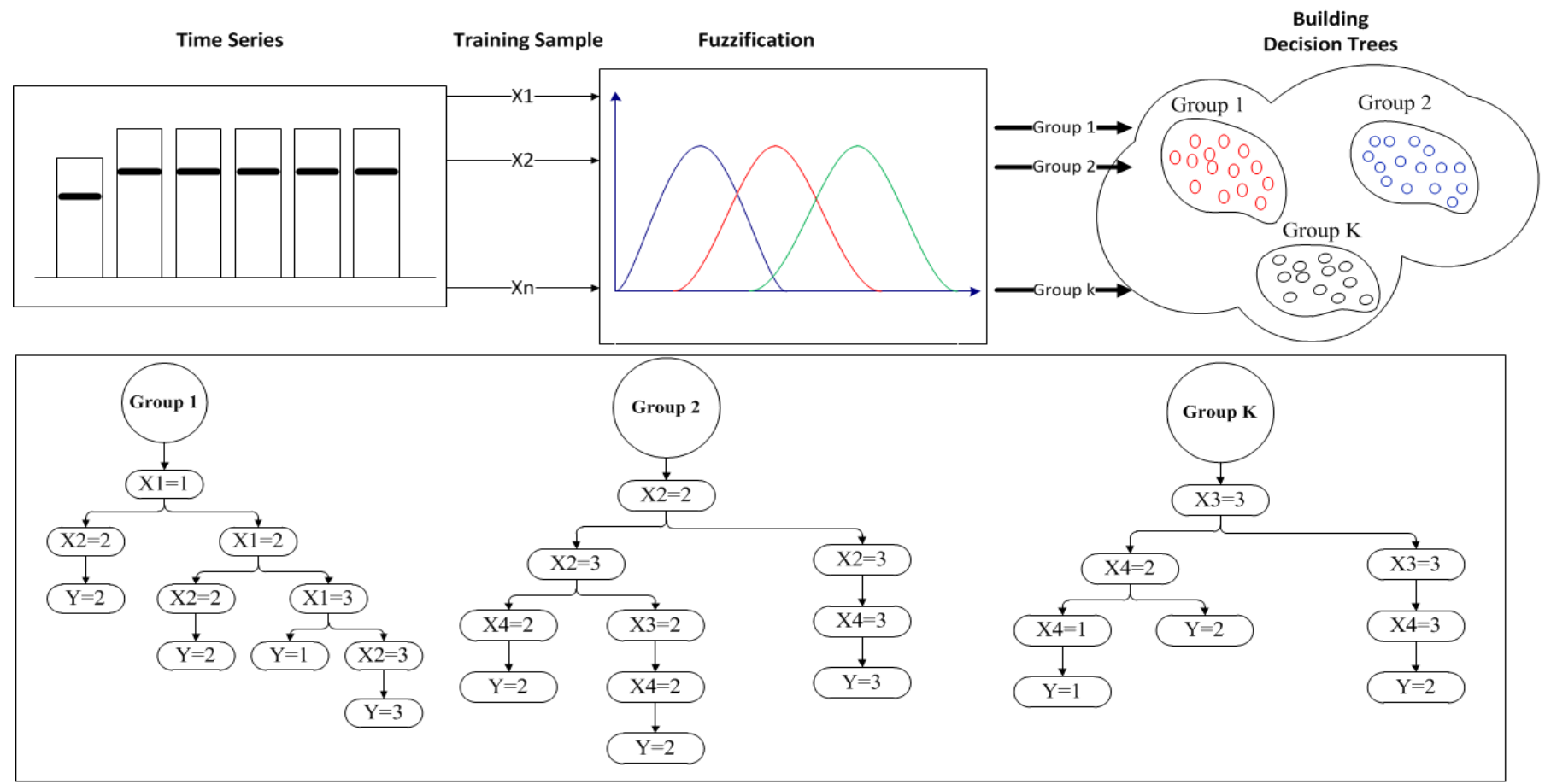

Fig. 2. DTFR process chart.

$C_{i}, i=1,2,3, \ldots, m$, , every category number are presented in

freg $\left(C_{i}, S\right),|S|$ means all values in data set $S$. The probability that every category data appears can be calculated as follows:

$$
\frac{\operatorname{freg}\left(C_{i}, S\right)}{|S|}
$$

Then according to information theory, the function, which measures the amount of information of occurrence of receiving the symbol $S$, is defined as follows:

$$
-\log _{2} \frac{\operatorname{freg}\left(C_{i}, S\right)}{|S|}
$$

Then we multiply the probability that every data appears with the information gain that every categorized data set can get except training data set $S$. The entropy of data set $S$ having symbols $s_{i}$ and probability $p_{i}$ is calculated as follows:

$$
I\left(s_{1}, s_{2}, \ldots, s_{m}\right)=-\sum_{i=1}^{m} p_{i} \log _{2}\left(p_{i}\right)
$$

Assume set $A$ can be split into sub-set $s_{1}, s_{2}, \ldots, s_{m}$, then we understand that splitting information equal to sub-set information multiplied by every sub-set proportion amount. The entropy of set $A$ is calculated follows: rules are described in Fig. 2.

The detailed procedures of ID3 decision tree is described in algorithm 3 .

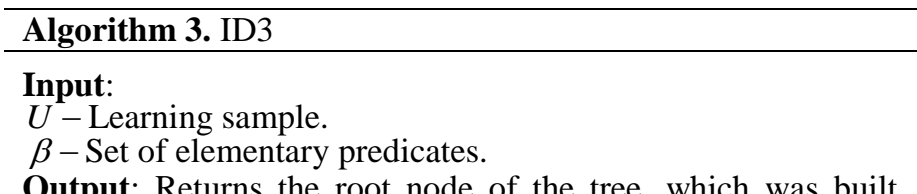

Output: Returns the root node of the tree, which was built based on a sample $U$;

1. PROCEDURE LearnID3 $(U)$.

2. If all objects of ( $U$ ) belong to the same class $c \in Y$

3. Create a new $v$

where $v-$ the leaf of tree in which each internal vertex $v \in V$

4. $c_{v}=c$

5. RETURN $(v)$.

6. find the predicate with the maximum information:

$$
\beta=\arg \max _{\beta \in B} I(\beta, U)
$$

7. Divide the learning sample into two parts $U=U_{0} \cup U_{1}$ by the predicate $\beta$ :

8. If $U_{0}=0$ or $U_{1}=0$

$$
\begin{aligned}
& U_{0}:=\{x \in U: \beta(x)=0\} ; \\
& U_{1}:=\{x \in U: \beta(x)=1\} ;
\end{aligned}
$$

10. Create a new leaf $v$.

11. $c_{v}=$ class, in which the most of the objects $U$. 

12. ELSE
13. $\beta_{v}=\beta$.
14. $L_{v}:=$ LearnID3 $\left(U_{0}\right)$. (build a left sub tree)
15. $R_{v}:=$ LearnID3 $\left(U_{1}\right)$. (build a right sub tree)
16. RETURN $v$.

\section{EXPERIMENTAL RESULT}

A. Computational experiment for electric power consumption

The information base for computing experiment for energy consumption is historical data about the consumption of the building in the previous month including 1 time series. The number of members in the time series for the month is 2688 time points. Below are presented the results of a computational experiment method of building decision trees based on fuzzy rules, as well as the quality of the classification decision tree using analytical platform "Daductor" [27]

Calculating experiment results obtained with the following parameters:

TABLE I. PARAMETERS OF THE COMPUTATIONAL EXPERIMENT

\begin{tabular}{lc}
\multicolumn{1}{c}{ Parameters } & Number \\
\hline Fuzzy Sets $A_{j}$ & 4 \\
Width of Gaussian membership functions $\sigma_{j}$ & 10 \\
Autoregressive variable & 4 \\
Forecasting horizon $\lambda$ & 96 \\
\hline
\end{tabular}

In the process of constructing fuzzy decision tree obtained four rules presented in Table 2 with different certainties. In table 7 and Fig. 3 shown the results of quality classification FKB $80 \%$ correctly classified classes and $20 \%$ incorrectly classified classes.

\begin{tabular}{lllll}
3.0 & 1.0 & 1.0 & 1.0 & 1.0 \\
2.0 & 2.0 & 2.0 & 1.0 & 1.0 \\
2.0 & 2.0 & 1.0 & 1.0 & 1.0 \\
\hline
\end{tabular}

TABLE IV. LAERNING SAMPLE 2 WITH CERTAINTY $66.66 \%$, NUMBER OF RULE $Y_{n}=2$, SUPPORT $Y_{\text {sup }}=20.68 \%$

\begin{tabular}{ccccc}
\hline $\mathbf{X 1}$ & $\mathbf{X} \mathbf{2}$ & $\mathbf{X 3}$ & $\boldsymbol{X 4}$ & $\boldsymbol{Y}$ \\
\hline 1.0 & 1.0 & 1.0 & 2.0 & 3.0 \\
1.0 & 2.0 & 3.0 & 2.0 & 4.0 \\
4.0 & 4.0 & 4.0 & 2.0 & 2.0 \\
4.0 & 4.0 & 2.0 & 2.0 & 4.0 \\
1.0 & 1.0 & 2.0 & 2.0 & 3.0 \\
4.0 & 4.0 & 3.0 & 2.0 & 2.0 \\
4.0 & 3.0 & 2.0 & 2.0 & 2.0 \\
3.0 & 2.0 & 2.0 & 2.0 & 2.0 \\
2.0 & 2.0 & 2.0 & 2.0 & 2.0 \\
1.0 & 2.0 & 2.0 & 2.0 & 2.0 \\
2.0 & 2.0 & 1.0 & 2.0 & 2.0 \\
2.0 & 1.0 & 2.0 & 2.0 & 2.0 \\
\hline
\end{tabular}

TABLE V. LAERNING SAMPLE 3 WITH CERTAINTY $84.21 \%$, NUMBER OF RULE $Y_{n}=4$, SUPPORT $Y_{\text {sup }}=32.75 \%$

\begin{tabular}{ccccc}
\hline $\mathbf{X 1}$ & $\mathbf{X} 2$ & $\mathbf{X 3}$ & $\boldsymbol{X} \mathbf{H}$ & $\boldsymbol{Y}$ \\
\hline 2.0 & 3.0 & 2.0 & 4.0 & 3.0 \\
2.0 & 4.0 & 3.0 & 4.0 & 4.0 \\
4.0 & 3.0 & 4.0 & 4.0 & 4.0 \\
3.0 & 4.0 & 4.0 & 4.0 & 4.0 \\
4.0 & 4.0 & 4.0 & 4.0 & 4.0 \\
4.0 & 2.0 & 2.0 & 4.0 & 4.0 \\
2.0 & 2.0 & 4.0 & 4.0 & 4.0 \\
2.0 & 4.0 & 4.0 & 4.0 & 4.0 \\
3.0 & 3.0 & 3.0 & 4.0 & 4.0 \\
3.0 & 3.0 & 4.0 & 4.0 & 4.0 \\
1.0 & 2.0 & 4.0 & 4.0 & 4.0 \\
\hline
\end{tabular}

TABLE II. RULES OF DECISION TREE

\begin{tabular}{|c|c|c|c|c|c|c|}
\hline \multirow{2}{*}{ № } & \multirow{2}{*}{ Condition } & \multirow{2}{*}{ Consequence } & \multicolumn{2}{|c|}{ Support } & \multicolumn{2}{|c|}{ Certainty } \\
\hline & & & $\%$ & Number & $\%$ & Number \\
\hline 1 & $\mathrm{X} 4<1.5$ & 1 & 18.97 & 11 & 100.00 & 11 \\
\hline 2 & $X 4>=1.5 \& X 4<2.5$ & 2 & 20.69 & 12 & 66.67 & 8 \\
\hline 3 & $\mathrm{X} 4>=1.5 \& \mathrm{X} 4>=2.5 \& \mathrm{X} 4<3$ & 3 & 27.59 & 16 & 68.75 & 11 \\
\hline 4 & $X 4>=1.5 \& X 4>=2.5 \& X 4>=3.5$ & 4 & 32.76 & 19 & 84.21 & 16 \\
\hline
\end{tabular}

TABLE III. LAERNING SAMPLE 1 WITH CERTAINTY 100\%, NUMBER OF RULE $Y_{n}=1$, SUPPORT $Y_{\text {sup }}=18.96 \%$

\begin{tabular}{ccccc}
\hline $\mathbf{X 1}$ & $\mathbf{X 2}$ & $\mathbf{X 3}$ & $\boldsymbol{X 4}$ & $\boldsymbol{Y}$ \\
\hline 1.0 & 1.0 & 1.0 & 1.0 & 1.0 \\
4.0 & 4.0 & 2.0 & 1.0 & 1.0 \\
4.0 & 2.0 & 1.0 & 1.0 & 1.0 \\
2.0 & 1.0 & 1.0 & 1.0 & 1.0 \\
4.0 & 4.0 & 4.0 & 1.0 & 1.0 \\
4.0 & 4.0 & 1.0 & 1.0 & 1.0 \\
4.0 & 1.0 & 1.0 & 1.0 & 1.0 \\
4.0 & 4.0 & 3.0 & 1.0 & 1.0 \\
4.0 & 3.0 & 1.0 & 1.0 & 1.0 \\
\hline
\end{tabular}

TABLE VI. LAERNING SAMPLE 4 WITH CERTAINTY $84.21 \%$, NUMBER OF RULE $Y_{n}=4$, SUPPORT $Y_{\text {sup }}=32.75 \%$

\begin{tabular}{ccccc}
\hline $\mathbf{X 1}$ & $\mathbf{X 2}$ & $\mathbf{X 3}$ & $\boldsymbol{X 4}$ & $\boldsymbol{Y}$ \\
\hline 2.0 & 3.0 & 2.0 & 4.0 & 3.0 \\
2.0 & 4.0 & 3.0 & 4.0 & 4.0 \\
4.0 & 3.0 & 4.0 & 4.0 & 4.0 \\
3.0 & 4.0 & 4.0 & 4.0 & 4.0 \\
4.0 & 4.0 & 4.0 & 4.0 & 4.0 \\
4.0 & 2.0 & 2.0 & 4.0 & 4.0 \\
2.0 & 2.0 & 4.0 & 4.0 & 4.0 \\
2.0 & 4.0 & 4.0 & 4.0 & 4.0 \\
\hline
\end{tabular}




\begin{tabular}{lllll}
3.0 & 3.0 & 3.0 & 4.0 & 4.0 \\
3.0 & 3.0 & 4.0 & 4.0 & 4.0 \\
1.0 & 1.0 & 2.0 & 4.0 & 4.0 \\
1.0 & 2.0 & 4.0 & 4.0 & 4.0 \\
\hline
\end{tabular}

TABLE VII. Results OF QuAlity Classification FuZZY DECISION TREE

\begin{tabular}{|l|c|c|c|c|c|}
\hline \multirow{2}{*}{ Actual } & \multicolumn{5}{|c|}{ Classified } \\
\cline { 2 - 6 } & 1 & 2 & 3 & 4 & Sum \\
\hline 1 & 12 & & 1 & & 13 \\
\hline 2 & & 8 & 1 & & 9 \\
\hline 3 & & 2 & 13 & 3 & 18 \\
\hline 4 & & 2 & 3 & 16 & 21 \\
\hline Sum & 12 & 12 & 18 & 19 & 61 \\
\hline
\end{tabular}

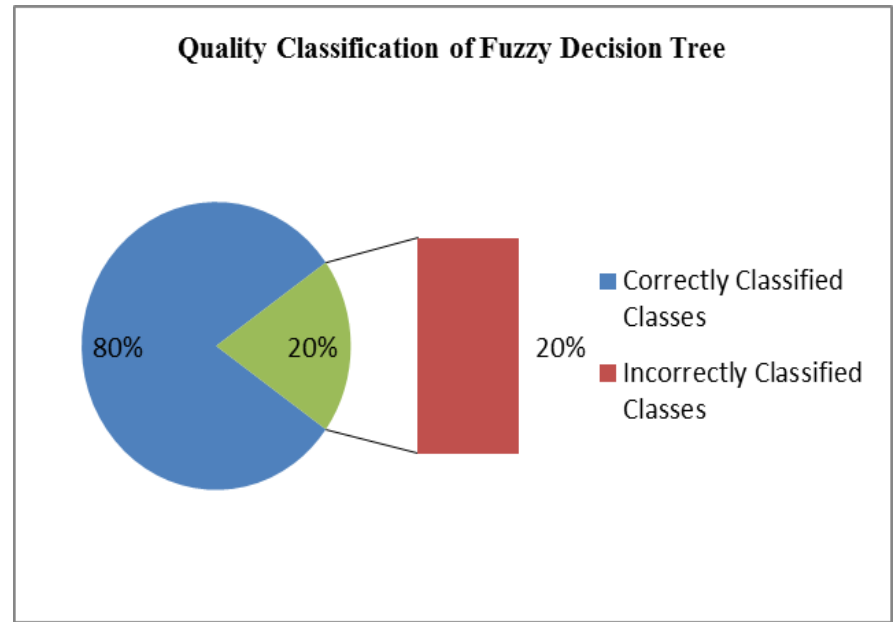

Fig. 3. Quality classification of fuzzy decision tree.

B. Computational experiment for Solar photovoltaic (PV) power generation

The information base for computing experiment for solar$\mathrm{PV}$ power generation is historical data about the solar-PV power generation (PG) in the previous five days including 1 time series. The numbers of members in the time series for the 5 days are 480 time points. Below are presented the results of a computational experiment method of building decision trees based on the fuzzy rules, as well as the quality of the classification decision tree using analytical platform "Daductor" [27].

Calculating experiment results obtained with the following parameters:

TABLE VIII. PARAMETERS OF THE COMPUTATIONAL EXPERIMENT

\begin{tabular}{lc}
\multicolumn{1}{c}{ Parameters } & Number \\
\hline Fuzzy Sets $A_{j}$ & 4 \\
Width of Gaussian membership functions $\sigma_{j}$ & 10 \\
Autoregressive variable & 4 \\
Forecasting horizon $\lambda$ & 96 \\
\hline
\end{tabular}

In the process of constructing fuzzy decision tree obtained 5 rules presented in Table 14 with different certainties. In table 15 and Fig. 4 shown the results of quality classification FKB $83 \%$ correctly classified classes and $17 \%$ incorrectly classified classes.

TABLE IX. LAERNING SAMPLE 1 WITH CERTAINTY 83.33\%, NUMBER OF RULE $Y_{n}=1$, SUPPORT $Y_{\text {sup }}=27.27 \%$

\begin{tabular}{lllll}
\hline $\mathbf{X 1}$ & $\mathbf{X} 2$ & $\mathbf{X 3}$ & $\boldsymbol{X} 4$ & $\boldsymbol{Y}$ \\
\hline 1.0 & 1.0 & 1.0 & 1.0 & 1.0 \\
2.0 & 3.0 & 2.0 & 1.0 & 1.0 \\
3.0 & 2.0 & 1.0 & 1.0 & 1.0 \\
2.0 & 1.0 & 1.0 & 1.0 & 2.0 \\
1.0 & 2.0 & 2.0 & 1.0 & 1.0 \\
2.0 & 2.0 & 1.0 & 1.0 & 1.0 \\
1.0 & 1.0 & 1.0 & 1.0 & 1.0 \\
2.0 & 3.0 & 2.0 & 1.0 & 1.0 \\
3.0 & 2.0 & 1.0 & 1.0 & 1.0 \\
2.0 & 1.0 & 1.0 & 1.0 & 2.0 \\
1.0 & 2.0 & 2.0 & 1.0 & 1.0 \\
2.0 & 2.0 & 1.0 & 1.0 & 1.0 \\
2.0 & 2.0 & 1.0 & 1.0 & 1.0 \\
\hline
\end{tabular}

TABLE X. LAERNING SAMPLE 2 WITH CERTAINTY $100 \%$, NUMBER OF RULE $Y_{n}=2$, SUPPORT $Y_{\text {sup }}=13.63 \%$

\begin{tabular}{lllll}
\hline $\mathbf{X 1}$ & $\mathbf{X} 2$ & $\mathbf{X 3}$ & $\boldsymbol{X 4}$ & $\boldsymbol{Y}$ \\
\hline 1.0 & 1.0 & 1.0 & 2.0 & 2.0 \\
1.0 & 1.0 & 2.0 & 2.0 & 2.0 \\
1.0 & 2.0 & 2.0 & 2.0 & 2.0 \\
\hline
\end{tabular}

TABLE XI. LAERNING SAMPLE 3 WITH CERTAINTY $71.42 \%$, NUMBER OF RULE $Y_{n}=3$, SUPPORT $Y_{\text {sup }}=31.81 \%$

\begin{tabular}{lllll}
\hline X1 & X2 & X3 & X4 & $\boldsymbol{Y}$ \\
\hline 2.0 & 2.0 & 2.0 & 2.0 & 3.0 \\
2.0 & 2.0 & 2.0 & 3.0 & 3.0 \\
2.0 & 2.0 & 3.0 & 3.0 & 3.0 \\
2.0 & 3.0 & 3.0 & 3.0 & 3.0 \\
3.0 & 3.0 & 3.0 & 3.0 & 3.0 \\
3.0 & 2.0 & 2.0 & 3.0 & 2.0 \\
2.0 & 2.0 & 3.0 & 2.0 & 1.0 \\
\hline
\end{tabular}

TABLE XII. LAERNING SAMPLE 4 WITH CERTAINTY $100 \%$, NUMBER OF RULE $Y_{n}=4$, SUPPORT $Y_{\text {sup }}=9.09 \%$

\begin{tabular}{lllll}
\hline $\mathbf{X 1}$ & $\mathbf{X} 2$ & $\mathbf{X 3}$ & $\boldsymbol{X 4}$ & $\boldsymbol{Y}$ \\
\hline 4.0 & 4.0 & 4.0 & 3.0 & 2.0 \\
4.0 & 4.0 & 3.0 & 2.0 & 2.0 \\
4.0 & 3.0 & 2.0 & 2.0 & 3.0 \\
\hline
\end{tabular}


TABLE XIII. LAERNING SAMPLE 5 WITH CERTAINTY $100 \%$, NUMBER OF RULE $Y_{n}=5$, SUPPORT $Y_{\text {sup }}=18.18 \%$

\begin{tabular}{ccccc}
\hline $\mathbf{X 1}$ & $\mathbf{X 2}$ & $\mathbf{X 3}$ & $\mathbf{X 4}$ & $\boldsymbol{Y}$ \\
\hline 3.0 & 3.0 & 3.0 & 4.0 & 4.0 \\
3.0 & 3.0 & 4.0 & 4.0 & 4.0 \\
3.0 & 4.0 & 4.0 & 4.0 & 4.0 \\
4.0 & 4.0 & 4.0 & 4.0 & 4.0 \\
\hline
\end{tabular}

TABLE XIV. RULES Of DECISION TREE decision tree i.e. when formed a compact and small decision trees we can received the following results: i) easily interpretable and accurate classification decision trees, ii) facilitates the work of the decision-makers.

In this study, we have proposed a new method to construct fuzzy decision trees using fuzzy rule called (DTFR). The method of DTFR can provide automatically generate fuzzy sets and FKB. The results of computational experiment showed that the proposed DTFR exhibit the best performance in terms of accuracy and the size of the resulting tree.

\begin{tabular}{|c|c|c|c|c|c|c|}
\hline \multirow{2}{*}{ № } & \multirow{2}{*}{ Condition } & \multirow{2}{*}{ Consequence } & \multicolumn{2}{|c|}{ Support } & \multicolumn{2}{|c|}{ Certainty } \\
\hline & & & $\%$ & Number & $\%$ & Number \\
\hline 1 & $\mathrm{X} 4<3.5 \& \mathrm{X} 4<1.5$ & 1 & 27 & 6 & 83.33 & 5 \\
\hline 2 & $\mathrm{X} 4<3.5 \& \mathrm{X} 4>=1.5 \& \mathrm{X} 1<1.5$ & 2 & 13.64 & 3 & 100 & 3 \\
\hline 3 & $\mathrm{X} 4<3.5 \& \mathrm{X} 4>=1.5 \& \mathrm{X} 1>=1.5 \& \mathrm{X} 1<3.5$ & 3 & 31.82 & 7 & 71.43 & 5 \\
\hline 4 & $\mathrm{X} 4\langle 3.5 \& \mathrm{X} 4>=1.5 \& \mathrm{X} 1>=1.5 \& \mathrm{X} 1\rangle=3.5$ & 2 & 9.09 & 2 & 100 & 2 \\
\hline
\end{tabular}

TABLE XV.

DECISION TREE

\begin{tabular}{|l|c|c|c|c|c|}
\hline & \multicolumn{5}{|c|}{ Classified } \\
\hline Actual & 1 & 2 & 3 & 4 & Sum \\
\hline 1 & 5 & & 1 & & 6 \\
\hline 2 & 1 & 5 & 1 & & 7 \\
\hline 3 & & 1 & 5 & & 6 \\
\hline 4 & & & & 4 & 4 \\
\hline Sum & 6 & 6 & 7 & 4 & 23 \\
\hline
\end{tabular}

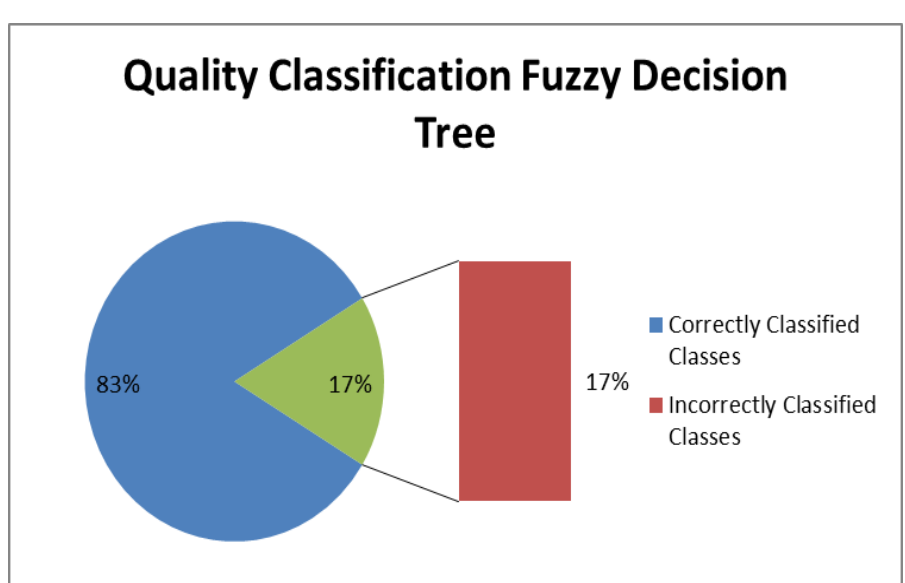

Fig. 4. Results of Quality classification of fuzzy decision tree.

\section{CONCLUSIONS}

The results obtained during execution of the experiment depend on the number of fuzzy sets (FS) i.e. if we will automatically set the number of FS ascending the size of FKB will be raising. In the theory of fuzzy sets and decision trees proved that one of the important indicators of effective FKB is reducing the number of fuzzy segments. The size of the FKB affects the efficiency, interpretability and the size of the

\section{ACKNOWLEDGMENTS}

The reported study was partially supported by RFBR, research project No. 16-37-60066 and MD-6964.2016.9.

\section{REFERENCES}

Y. S. T. Lim, W. Loh, "An Empirical Comparison of Decision Trees and Other Classification Methods," Tech. Report, Dep. Stat. Univ. Wisconsin, 1997.

J. Gehrke, V. Ganti, R. Ramakrishnan, and W. Y. Loh, "BOAT Optimistic Decision Tree Construction," SIGMOD Rec. (ACM Spec. Interes. Gr. Manag. Data), vol. 28, no. 2, pp. 169-180, 1999.

N. Manwani and P. S. Sastry, "Geometric decision tree.," IEEE Trans. Syst. Man. Cybern. B. Cybern., vol. 42, no. 1, pp. 181-92, Feb. 2012.

M. Mehta, R. Agrawal, and J. Rissanen, "SLIQ: A fast scalable classifier for data mining," in Lecture Notes in Computer Science (including subseries Lecture Notes in Artificial Intelligence and Lecture Notes in Bioinformatics), 1996, vol. 1057 LNCS, pp. 18-32.

[5] L. W. Olaru, Cristina, "A complete fuzzy decision tree technique," Fuzzy Sets Syst., vol. 140, no. 3, pp. 563-565.

[6] B. A. Badr S, "Case study of inaccuracies in the granulation of decision trees," Soft Comput 15, pp. 1129-1136, 2011.

[7] W. P. Ayaz Isazadeh, Farnaz Mahan, "MFlexDT: multi flexible fuzzy decision tree for data stream classification," Soft Comput., pp. $1-15,2012$.

[8] J. R. Quinlan, "Induction of decision trees," Mach. Learn., vol. 1, no. 1, pp. 81-106, Mar. 1986.

[9] C. Z. Janikow, "Fuzzy decision trees: issues and methods," IEEE Trans. Syst. Man, Cybern. Part B, vol. 28, no. 1, pp. 1 - 14, 1998.

[10] M. J. S. Yuan, Yufei, "Induction of fuzzy decision trees," vol. 69, no. 2, pp. $125-139,1995$.

[11] M. S. Liam Evans, Niels Lohse, "A fuzzy-decision-tree approach for manufacturing technology selection exploiting experience-based information," Expert Syst. Appl., vol. 40, no. 16, 2013.

[12] C. Z. Janikow, "FID4.1: an overview," Fuzzy Information, 2004. Process. NAFIPS '04. IEEE Annu. Meet., vol. 2, pp. 877 - 881, 
2004.

[13] L. Olaru, C., Wehenkel, "A complete fuzzy decision tree technique," Fuzzy Sets Syst., vol. 138, no. 2, pp. 221-254, 2003.

[14] J.-L. L. Pei-Chann Chang, Chin-Yuan Fan, "Trend discovery in financial time series data using a case based fuzzy decision tree," vol. 38, no. 5, pp. 6070-6080, 1987.

[15] M. Khan, U., Shin, H., Choi, J.P. and Kim, "wFDT - Weighted Fuzzy Decision Trees for Prognosis of Breast Cancer Survivability," Proc. Seventh Australas. Data Min. Conf. (AusDM 2008), Glenelg, South Aust. CRPIT, vol. 87, pp. 141-152, 2008.

[16] L. Boyen, Xavier and Wehenkel, "Automatic induction of fuzzy decision trees and its application to power system security assessment," Fuzzy Sets Syst., vol. 102, pp. 3-19, 19999.

[17] L. Z.; Z. S. W. Meizhen, "Fuzzy Decision Tree Based Inference Technology for Spam Behavior Recognition,” pp. 287-300, 2013.

[18] C. J. S. L. Breiman, J.H.Friedman, R.A.Olshen, "Classification and Regression Trees,” Wadsworth Int. Group, Belmont, Calif., 1984

[19] P. S. S. S. Shah, "New algorithms for learning and pruning oblique decision trees," pp. $494-505,2013$.

[20] G. L. Kovács, I. Mezgár, S. Kopácsi, D. Gavalcová, and J. Nacsa, "Application of artificial intelligence to problems in advanced manufacturing systems," Comput. Integr. Manuf. Syst., vol. 7, no. 3, pp. 153-160, 1994.

[21] S.-J. Chuu, "Selecting the advanced manufacturing technology using fuzzy multiple attributes group decision making with multiple fuzzy information," Comput. Ind. Eng., vol. 57, no. 3, pp. 10331042,2009

[22] J. Zhao, M.-H. Chen, Y.-Z. Liu, D.-R. Li, "The application of optimized fuzzy decision trees in business intelligence," Int. Conf. Mach. Learn. Cybern. Dalian; China, pp. 2212-2217, 2006.

[23] M. A. Al-Gunaid, "NEURO-FUZZY MODEL SHORT TERM FORECASTING OF ENERGY CONSUMPTION," PRIKASPIJSKIJ ZhURNAL Upr. I Vysok. Tehnol., vol. 2, pp. 47$56,2013$.

[24] R. K. Lai, C.-Y. Fan, W.-H. Huang, and P.-C. Chang, "Evolving and clustering fuzzy decision tree for financial time series data forecasting," Expert Syst. Appl., vol. 36, no. 2, pp. 3761-3773, Mar. 2009

[25] M. A. M.V. Shcherbakov, Al-Gunaid, "SHORT-TERM FORECASTING OF ENERGY CONSUMPTION USING FUZZY NEURAL NETWORK," NAUChNYE Tr. SWORLD, vol. 4, p. 5355, 2012.

[26] M. A. M.V. Shcherbakov, Al-Gunaid, "Development and Analysis of Hybrid Fuzzy Model Identification," Izv. Volgogr. Gos. Teh. Univ., vol. 15, pp. 66-72, 2012.

[27] BaseGroup, "Analytical Platform 'Daductor," BaseGroup Labs, 2016. [Online]. Available: https://basegroup.ru/deductor/description. 
